PROCEEDINGS OF THE

AMERICAN MATHEMATICAL SOCIETY

Volume 129, Number 7, Pages 2175-2185

S 0002-9939(00)05775-0

Article electronically published on December 4, 2000

\title{
VOL3 AND OTHER EXCEPTIONAL HYPERBOLIC 3-MANIFOLDS
}

\author{
K. N. JONES AND A. W. REID
}

(Communicated by Ronald A. Fintushel)

\begin{abstract}
D. Gabai, R. Meyerhoff and N. Thurston identified seven families of exceptional hyperbolic manifolds in their proof that a manifold which is homotopy equivalent to a hyperbolic manifold is hyperbolic. These families are each conjectured to consist of a single manifold. In fact, an important point in their argument depends on this conjecture holding for one particular exceptional family. In this paper, we prove the conjecture for that particular family, showing that the manifold known as Vol3 in the literature covers no other manifold. We also indicate techniques likely to prove this conjecture for five of the other six families.
\end{abstract}

\section{INTRODUCTION}

The following important rigidity theorem is proved by D. Gabai, R. Meyerhoff and N. Thurston in [6]:

Theorem 1.1. Let $N$ be a closed hyperbolic 3-manifold, $M$ a closed irreducible 3-manifold and $f: M \rightarrow N$ a homotopy equivalence. Then $f$ is homotopic to a homeomorphism.

This theorem is proved by a rigorous computer-assisted procedure, which has its origins in an earlier work of Gabai [5], where the above theorem was proven with an added technical condition concerning the existence of a certain kind of closed geodesic (one which satisfies the "insulator condition" defined in [5]) in $N$. The proof of Theorem 1.1 is achieved by using several computer programs to show that all closed hyperbolic 3-manifolds, apart from seven exceptional families, possess such a closed geodesic. The focus of this note is these seven families. It is conjectured that these seven families simply consist of seven manifolds. The main aim for us is to use arithmetic techniques to prove uniqueness for one of these families (denoted $X_{0}$ in [6]), and investigate some properties. To state the theorem we recall some notation from [6], and the relation to traces that we shall use.

Received by the editors April 19, 1999 and, in revised form, October 13, 1999 and November 8, 1999.

2000 Mathematics Subject Classification. Primary 57M50.

Key words and phrases. Hyperbolic 3-manifold, arithmetic manifold, homotopy hyperbolic 3-manifold.

The first author was partially supported by Ball State University.

The second author was partially supported by the Royal Society, NSF, the A. P. Sloan Foundation and a grant from the Texas Advanced Research Program.

(C)2000 American Mathematical Society 
The exceptional manifolds of interest in [6] are specified by a triple $\left(L^{\prime}, D^{\prime}, R^{\prime}\right)$ of approximate complex numbers representing exponentials of various complex lengths which suffice to geometrically describe a conjugacy class of 2 -generator subgroups (generated by $f$ and $w$ in the notation of [6]) of $\operatorname{PGL}(2, \mathbf{C})$.

Such a 2-generator group is completely specified up to conjugacy by a different triple of complex numbers, namely, the normalized traces (that is, the traces of the $\mathrm{SL}(2, \mathbf{C})$ representatives) of the two generators and their product. In general the traces themselves are not necessarily in the invariant trace-field (see [10] and $\S 2$ below) but the traces of the squares of those generators are. Furthermore, it is an easy matter to take "square roots" in $\operatorname{PGL}(2, \mathbf{C})$ since, if $x$ has determinant one, $(x \pm I)^{2}=(\operatorname{tr} x \pm 2) x$ which is equal to $x$ in $\operatorname{PGL}(2, \mathbf{C})$. Hence, one may easily (though not uniquely) recover the triple of traces of $\langle x, y\rangle$ from that of $\left\langle x^{2}, y^{2}\right\rangle$, as follows:

$$
\begin{aligned}
\operatorname{tr} x & =\sqrt{2 \pm \operatorname{tr} x^{2}} \\
\operatorname{tr} y & =\sqrt{2 \pm \operatorname{tr} y^{2}} \\
\operatorname{tr} x y & =\left(\operatorname{tr} x^{2} y^{2}+\operatorname{tr} x^{2}+\operatorname{tr} y^{2}\right) /(\operatorname{tr} x \operatorname{tr} y)
\end{aligned}
$$

Therefore, it is most convenient from the arithmetic point of view to work with the triple

$$
\left(\operatorname{tr} f^{2}, \operatorname{tr} w^{2}, \operatorname{tr} f^{2} w^{2}\right)
$$

instead of the original $\left(L^{\prime}, D^{\prime}, R^{\prime}\right)$ triple. We call this the trace triple associated to $\langle f, w\rangle$.

Note that, a priori, there are four possible conjugacy classes of two-generator subgroups corresponding to a given conjugacy class of two-generator subgroup generated by squares. However, using the particular correspondence detailed below, it is easy to check that the original generators correspond to those obtained by using both positive signs in the formulae above, so that the original generators $f$ and $w$ are scalar multiples of $f^{2}+I$ and $w^{2}+I$ respectively.

The correspondence between the triple of [6] and ours is given by

$$
\begin{aligned}
\operatorname{tr} f^{2} & =L^{\prime}+\frac{1}{L^{\prime}}, \\
\operatorname{tr} w^{2} & =\frac{\left(R^{\prime}+\frac{1}{R^{\prime}}+2\right)\left(D^{\prime}+\frac{1}{D^{\prime}}+2\right)-8}{4}, \\
\operatorname{tr} f^{2} w^{2} & =\frac{\left(D^{\prime}+\frac{1}{D^{\prime}}+2\right)\left(R^{\prime} L^{\prime}+\frac{1}{R^{\prime} L^{\prime}}\right)+\left(D^{\prime}+\frac{1}{D^{\prime}}-2\right)\left(L^{\prime}+\frac{1}{L^{\prime}}\right)}{4} .
\end{aligned}
$$

With this notation, we prove (see Experimental Theorem 1.30, Proposition 3.1 and Remark 3.3 of [6] ):

Theorem 1.2. The triple $\left(L^{\prime}, D^{\prime}, R^{\prime}\right)$ associated to $X_{0}$ occurs for a unique closed hyperbolic 3-manifold $M$ of volume $v_{0}$, the volume of the regular ideal simplex in $\mathbf{H}^{3}$. The relevant trace triple is $(-1-\sqrt{-3},-1-\sqrt{-3},-1+\sqrt{-3})$.

The main application of Theorem 1.2 (see Theorem 3.1 below) to [6] is that the manifold $M$ does not cover any other hyperbolic 3-manifold (see Proposition 3.1 of [6]). The manifold $M$ is what has been called Vol3 in the literature (see $\S 3$ ).

We also discuss five of the other exceptional manifolds. 


\section{Arithmetic PRELiminaries}

Here we recall the definition of an arithmetic Kleinian group as well as discuss some of Borel's work [2] on maximal elements in the commensurability class of an arithmetic Kleinian group.

2.1. Arithmetic Kleinian groups. Let $k$ be a field of characteristic different from 2. The standard notation for a quaternion algebra over $\mathrm{k}$ is the following: let $a$ and $b$ be non-zero elements of $k$. Then $\left(\frac{a, b}{k}\right)$ denotes the quaternion algebra over $k$ with basis $\{1, i, j, i j\}$ subject to $i^{2}=a, j^{2}=b$ and $i j=-j i$. $\left(\frac{a, b}{k}\right)$ is called a Hilbert Symbol for the quaternion algebra.

If now $k$ is a number field, and $\nu$ a place of $k$, we say a quaternion algebra $A$ over $k$ is ramified at $\nu$ if $A \otimes_{k} k_{\nu}$ is a division algebra of quaternions. In the case when $\nu$ is associated to a real embedding of $k, A$ is ramified at $\nu$ if $A \otimes_{k} k_{\nu} \cong \mathcal{H}$ where $\mathcal{H}$ is the Hamiltonian quaternions over $\mathbf{R}$. We shall denote the set of places (resp. finite places) of $k$ at which $A$ is ramified by $\operatorname{Ram}(A)\left(\operatorname{resp} \cdot \operatorname{Ram}_{f}(A)\right)$.

Let $\Gamma$ be a Kleinian group and let $\mathbf{Q}(\operatorname{tr} \Gamma)$ denote the trace-field of $\Gamma$. When $\Gamma$ has finite co-volume $\mathbf{Q}(\operatorname{tr} \Gamma)$ is a finite extension of $\mathbf{Q}$. Following [1] and [10] we define the invariant trace-field $k \Gamma$ and invariant quaternion algebra $A \Gamma$ of $\Gamma$ as follows: let $\Gamma^{(2)}=\operatorname{gp}\left\{\gamma^{2}: \gamma \in \Gamma\right\}$. Then $k \Gamma=\mathbf{Q}\left(\operatorname{tr} \Gamma^{(2)}\right)$ and $A \Gamma$ is the quaternion algebra over $k \Gamma$ defined by (see [1])

$$
\mathrm{A} \Gamma=\left\{\sum a_{i} \gamma_{i}: a_{i} \in k \Gamma, \gamma_{i} \in \Gamma^{(2)}\right\}
$$

where all sums are finite. $k \Gamma$ and $\mathrm{A} \Gamma$ are invariants of the commensurability class of $\Gamma$. Now $A \Gamma$ can be explicitly determined from $\Gamma$ (see [7]):

Lemma 2.1. Let $\Gamma$ be a co-compact Kleinian group for which $k \Gamma=\mathbf{Q}(\operatorname{tr} \Gamma)$, and let $\gamma$ and $\delta$ be a pair of non-commuting elements of $\Gamma$ with order different from 2 . Then,

$$
\mathrm{A} \Gamma \cong\left(\frac{\left(\operatorname{tr}^{2}(\gamma)-4\right),(\operatorname{tr}([\gamma, \delta])-2)}{k \Gamma}\right)
$$

We now recall the definition of arithmetic Kleinian groups (see 2], or [13] for details).

Let $k$ be a number field having exactly one complex place. Let $B$ be a quaternion algebra over $k$ which ramifies at all real places of $k$. Let $\mathcal{O}$ be an order of $B$ and let $\mathcal{O}^{1}$ be the group of elements of reduced norm 1 in $\mathcal{O}$. Over an embedding $k \hookrightarrow \mathbf{C}$ inducing the complex place of $k$ one may choose an algebra embedding $\rho: B \hookrightarrow M(2, \mathbf{C})$ which restricts to an injection $\rho: \mathcal{O}^{1} \hookrightarrow \mathrm{SL}(2, \mathbf{C})$. Let $\mathrm{P}$ : $\mathrm{SL}(2, \mathbf{C}) \rightarrow \operatorname{PSL}(2, \mathbf{C})$ be the natural projection. Then $\mathrm{P} \rho\left(\mathcal{O}^{1}\right)$ is a Kleinian group of finite co-volume. An arithmetic Kleinian group $\Gamma$ is a subgroup of $\operatorname{PSL}(2, \mathbf{C})$ commensurable with a group of the type $\mathrm{P} \rho\left(\mathcal{O}^{1}\right)$. We say $\Gamma$ is derived from $a$ quaternion algebra if $\Gamma$ is actually a subgroup of some $\mathrm{P} \rho\left(\mathcal{O}^{1}\right)$. We call $Q=\mathbf{H}^{3} / \Gamma$ arithmetic or derived from a quaternion algebra if $\Gamma$ is arithmetic or derived from a quaternion algebra.

It is shown in 9 ] that a Kleinian group $\Gamma$ of finite co-volume is arithmetic if and only if the group $\Gamma^{(2)}$ is derived from a quaternion algebra. 
2.2. Maximal arithmetic Kleinian groups. To describe Borel's results 2 we fix the following notation: let $B$ be a quaternion algebra over a number field $k$ with one complex place. Let $R_{k}$ denote the ring of integers of $k$. We shall use the notation $\bar{x}$ to denote an element of $B^{*} / k^{*}$ represented by $x \in B^{*}$.

A Kleinian group $\Gamma \subset \operatorname{PGL}(2, \mathbf{C})$ is maximal if it is maximal, with respect to inclusion, within its commensurability class. Borel [2] proved that any maximal arithmetic Kleinian group is isomorphic to some group $\Gamma_{S, \mathcal{O}}$, which we now define. Let $\mathcal{O}$ be a maximal order of $B$ and $S$ a finite (possibly empty) set of primes of $k$ disjoint from $\operatorname{Ram}_{f}(B)$. For each $\mathcal{P} \in S$ choose a local maximal order $E_{\mathcal{P}} \subset B_{\mathcal{P}}$ such that $\left[\mathcal{O}_{\mathcal{P}}: E_{\mathcal{P}} \cap \mathcal{O}_{\mathcal{P}}\right]=\operatorname{Norm}_{k / \mathbf{Q}}(\mathcal{P})$, where $\mathcal{O}_{\mathcal{P}}=\mathcal{O} \otimes_{R_{k}} R_{k_{\mathcal{P}}}$. We shall say that $x \in B_{\mathcal{P}}^{*}$ fixes $\mathcal{O}_{\mathcal{P}}$ (resp., $\left\{\mathcal{O}_{\mathcal{P}}, E_{\mathcal{P}}\right\}$ ) if $x \mathcal{O}_{\mathcal{P}} x^{-1}=\mathcal{O}_{\mathcal{P}}$ (resp., either $x$ fixes $\mathcal{O}_{\mathcal{P}}$ and $E_{\mathcal{P}}$, or $x \mathcal{O}_{\mathcal{P}} x^{-1}=E_{\mathcal{P}}$ and $\left.x E_{\mathcal{P}} x^{-1}=\mathcal{O}_{\mathcal{P}}\right)$. Borel's definition is

$\Gamma_{S, \mathcal{O}}=\left\{\bar{x} \in B^{*} / k^{*} \mid x\right.$ fixes $\mathcal{O}_{\mathcal{P}}$ for all $\mathcal{P} \notin S$, and for $\mathcal{P} \in S, x$ fixes $\left.\left\{\mathcal{O}_{\mathcal{P}}, E_{\mathcal{P}}\right\}\right\}$.

A specific maximal group that will be important to us has the following alternative description.

Let $\mathcal{O}$ be a maximal order of $B$ and let

$$
\Gamma_{\mathcal{O}}=\left\{\bar{x} \in B^{*} / k^{*} \mid x \mathcal{O} x^{-1}=\mathcal{O}\right\} .
$$

Via the complex place of $k$ we get an embedding $\rho: B \hookrightarrow M(2, \mathbf{C})$ and hence a $\bar{\rho}: B^{*} / k^{*} \hookrightarrow \operatorname{PGL}(2, \mathbf{C})$. For simplicity we identify $\Gamma_{\mathcal{O}}$ with $\bar{\rho}\left(\Gamma_{\mathcal{O}}\right)$. Then $\Gamma_{\mathcal{O}} \subset$ $\operatorname{PGL}(2, \mathbf{C})$ is an arithmetic Kleinian group giving rise to a hyperbolic 3-orbifold $\mathbf{H}^{3} / \Gamma_{\mathcal{O}}$ of finite volume. In the above description, if we take $S$ to be empty we find $\Gamma_{S, \mathcal{O}}=\Gamma_{\mathcal{O}}$ (see [2] for further details).

Two maximal orders $\mathcal{O}$ and $\mathcal{O}^{\prime}$ of $B$ are said to be of the same type if they are conjugate by an element of $B^{*}$. In this case $\Gamma_{S, \mathcal{O}}$ is conjugate to $\Gamma_{S, \mathcal{O}^{\prime}}$. Thus, to study all the $\Gamma_{S, \mathcal{O}}$ up to conjugacy, it suffices to select one $\mathcal{O}$ from each type. Types can be parametrized by the group $T_{B}$ defined as the group of fractional ideals of $k$, modulo the subgroup generated by squares of ideals, by ideals in $\operatorname{Ram}_{f}(B)$ and by principal ideals $(\alpha)$ generated by an $\alpha \in k^{*}$ which is positive at all real embeddings of $k$. The set of types is in bijection with the elements of $T_{B}$ [13] (recall that we are assuming that $\operatorname{Ram}(B)$ includes all real places).

2.3. Volume formula. We shall make use of a certain volume formula for arithmetic Kleinian groups (see [2] and [3], Prop. 2.1)

$$
\operatorname{Vol}\left(\mathbf{H}^{3} / \Gamma_{S, \mathcal{O}}\right)=\frac{2 \pi^{2} \zeta_{k}(2) d_{k}^{\frac{3}{2}}\left(\prod_{\mathcal{P} \in \operatorname{Ram}_{f}(B)} \frac{N \mathcal{P}-1}{2}\right) \prod_{\mathcal{P} \in S}(N \mathcal{P}+1)}{2^{m}\left(4 \pi^{2}\right)^{[k: \mathbf{Q}]}\left|T_{B}\right|}
$$

for some integer $m$ with $0 \leq m \leq|S|$. Here $\zeta_{k}$ denotes the Dedekind zeta function of $k, d_{k}$ is the absolute value of the discriminant of $k, \operatorname{Ram}_{f}(B)$ is the set of finite places of $k$ at which $A$ is ramified, $N$ denotes the absolute norm, and $S$ and $T_{B}$ are as defined above.

We remark that the case $S=\emptyset$ gives the minimal volume in the commensurability class.

Notation. If a prime ideal of the field $k$ has norm $n$, we denote this ideal by $\mathcal{P}_{n}$. 


\section{An ARITHMETIC MANIFOLD}

3.1. The computer generation of the manifold. We have written a suite of computer programs for investigating arithmetic hyperbolic 3-orbifolds which are capable (among other things) of calculating rigorously correct presentations of fundamental groups of these orbifolds. See [8] for a fuller discussion of these programs.

For our purposes, these programs may be regarded simply as a means of taking a group specified by a generating set of elements in a quaternion algebra over a number field with one complex place, ramified at all real places, and producing an approximate Dirichlet domain for a particular representation of this group into $\operatorname{PGL}(2, \mathbf{C})$ which has the property that a presentation of the group derived from the identification pattern on this approximate Dirichlet domain is guaranteed to be isomorphic to the original group. In addition, the presentation has the property that any torsion present in the original group will be recognizable as conjugate to a proper power relator in the presentation - this greatly simplifies the location of manifolds (as opposed to orbifolds) commensurable with the group.

To apply this machinery to the case of $X_{0}$, we proceed as follows (recall the discussion of $\$ 1$ ). Examining the data presented in [6] associated to $X_{0}$, we find that, at least to the precision specified there, the exceptional manifold being sought has fundamental group generated by $a^{2}+I$ and $b^{2}+I$ where

$$
\begin{aligned}
\operatorname{tr} a^{2} & =-1-\sqrt{-3}, \\
\operatorname{tr} b^{2} & =-1-\sqrt{-3} \\
\operatorname{tr} a^{2} b^{2} & =-1+\sqrt{-3} .
\end{aligned}
$$

Note that since the above traces are elements of $O_{3}$ (the ring of integers of $\mathbf{Q}(\sqrt{-3}))$ it is easy to see that the group $\left\langle a^{2}, b^{2}\right\rangle$ is discrete. Briefly,

$$
\left\{1, a^{2}, b^{2}, a^{2} b^{2}\right\}
$$

is an $O_{3}$-basis for an order $\mathcal{O}$ of the invariant quaternion algebra of $\left\langle a^{2}, b^{2}\right\rangle$ (see [4] or [8]) so that $\left\langle a^{2}, b^{2}\right\rangle$ is a subgroup of the arithmetic Kleinian group arising from $\mathcal{O}^{1}$; recall $\S 2$.

We now use the program to compute a presentation for the supergroup $\langle a, b\rangle$. This yields the presentation

$$
\left\langle a, b \mid b^{-1} a b a^{2} b a b^{-1} a^{2}, b a b^{-1} a b a b a^{-1} b a\right\rangle .
$$

This is the fundamental group of the manifold which is referred to as $M$ in the statement of Theorem 1.2 Arithmetic considerations (the invariant quaternion algebra is ramified at primes of norm 4 and 3, see below) lead us to the conclusion that $M$ is closed.

The program also computes the volume of this manifold as $1.0149 \ldots$ (correct to these five significant figures) and thus by arithmetic considerations detailed below the volume is exactly equal to $v_{0}$. We also readily calculate from this presentation that $H_{1}(M ; \mathbf{Z})=\mathbf{Z}_{3} \oplus \mathbf{Z}_{6}$.

This group is isomorphic to the presentation obtained in [11] for the fundamental group of a manifold given by a surgery description, namely, $(-1,2)-$ Dehn filling on a once-punctured torus bundle whose monodromy is $-R^{2} L$ in the usual $R L$ factorization. This presentation is

$$
\left\langle A, B \mid B^{2} A^{2} B^{2} A^{-1} B^{-1} A^{-1}, B^{-1} A B A^{3} B A B^{-1} A\right\rangle
$$


and the isomorphism between the two is given by $a=A B^{2}, b=A^{-1} B^{-1} A^{-2}$. In [12], it is shown that this manifold is arithmetic with volume $v_{0}$. The relevant traces for these generators are given by

$$
\begin{aligned}
\operatorname{tr} A^{2} & =-1+\sqrt{-3}, \\
\operatorname{tr} B^{2} & =(-5+\sqrt{-3}) / 2, \\
\operatorname{tr} A^{2} B^{2} & =(1-\sqrt{-3}) / 2 .
\end{aligned}
$$

Also using the program, we compute that the orbifold of minimal volume in the commensurability class of $M$ has the presentation

$$
\left\langle x, y, z \mid x^{2}=y^{2}=z^{2}=(x y z)^{4}=(x y x y x z)^{2}=(y z x z)^{2}=1\right\rangle
$$

for its fundamental group. We also compute that this orbifold has volume $0.1268 \ldots$ (correct to these four significant figures). An embedding of $\langle a, b\rangle$ into $\langle x, y, z\rangle$ is given by $a=x y x z x y z x y x y$ and $b=x y x z x y x z x$ (this is most readily checked by calculating traces in $\langle x, y, z\rangle)$.

Comparing the two (approximate) volumes readily gives an index of 8 for $\langle a, b\rangle$ in $\langle x, y, z\rangle$. Furthermore, we may define a homomorphism $\varphi$ from $\langle x, y, z\rangle$ onto the dihedral group of order 8 (given by $\left\langle s, t \mid s^{2}=t^{4}=1\right\rangle$ ) by $\varphi(x)=t^{2}, \varphi(y)=t s$, $\varphi(z)=s t^{2}$. One easily checks this to be a homomorphism since $\varphi(x y z)=t$, $\varphi(x y x y x z)=s$ and $\varphi(y z x z)=s t$. Further,

$$
\varphi(x y x z x y z x y x y)=\varphi(x y x z x y x z x)=1
$$

so that $\langle a, b\rangle$ is contained in the kernel of $\varphi$. Hence, since the index of $\langle a, b\rangle$ in $\langle x, y, z\rangle$ is 8 , we deduce that $\langle a, b\rangle$ coincides with the kernel of $\varphi$, and so is a normal subgroup of index 8 .

We also remark that $\langle x, y, z\rangle$ contains subgroups isomorphic to the dihedral group of order 8 generated, for example, by $y z x$ and $z$.

Figure 1 contains a labeled view of the computer-generated polyhedron which is the fundamental domain for $M$ with its face-pairing indicated. Figure 2 contains a perspective view (upper-half-space model) of the same polyhedron.

3.2. Arithmetic properties of the manifold. Here, we note that the algebra $A$ associated to the trace triple used for the construction of $M$ is ramified at two finite places: $\mathcal{P}_{3}$, associated to $\sqrt{-3}$, and $\mathcal{P}_{4}$, associated to 2 (which is inert).

To see this, we use the trace triple to calculate a Hilbert symbol

$$
\left(\frac{2 \sqrt{-3}-6,6 \sqrt{-3}-6}{\mathbf{Q}(\sqrt{-3})}\right)
$$

for $A$, and then observe that the norms of the upper Hilbert symbol entries are 48 and 144, respectively. This immediately rules out any finite ramification other than the two finite places indicated, since non-dyadic places which ramify an algebra must divide one of the entries of any Hilbert symbol for that algebra. To complete this calculation, we localize at the prime $\mathcal{P}_{3}$ and observe that the first entry is a uniformizer for the localized field, while the second is a non-square. Standard quaternion algebra techniques (see [13], for example) then show that $A$ is ramified at $\mathcal{P}_{3}$. Since the ramification set always has even cardinality, and there are no real places of this field, there must also be a dyadic prime which ramifies $A$, but $\mathcal{P}_{4}$ is the unique dyadic prime in this field. 


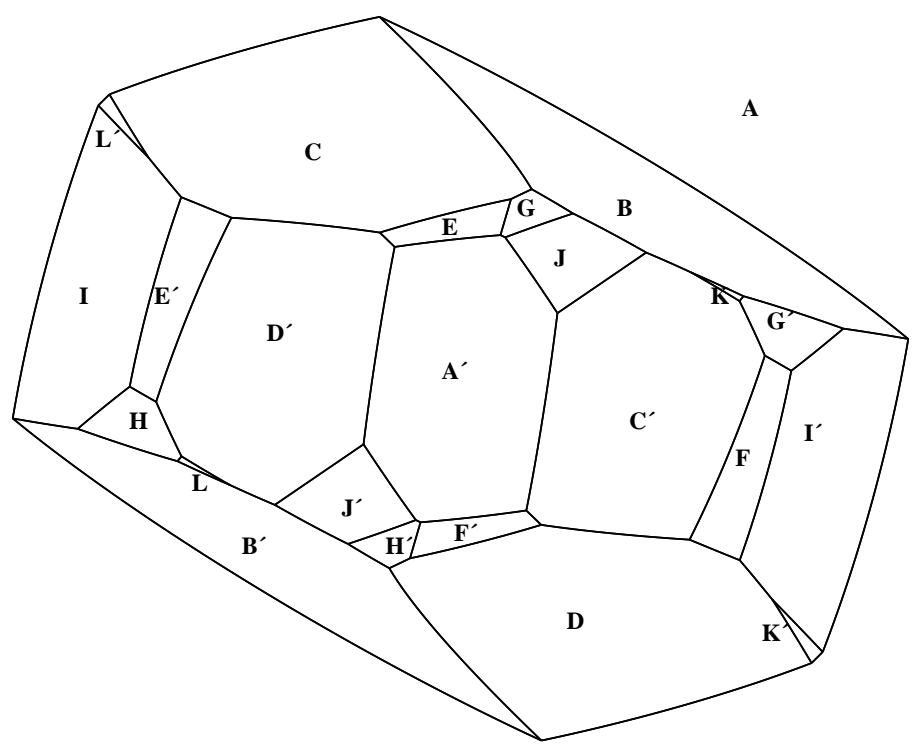

FIGURE 1.

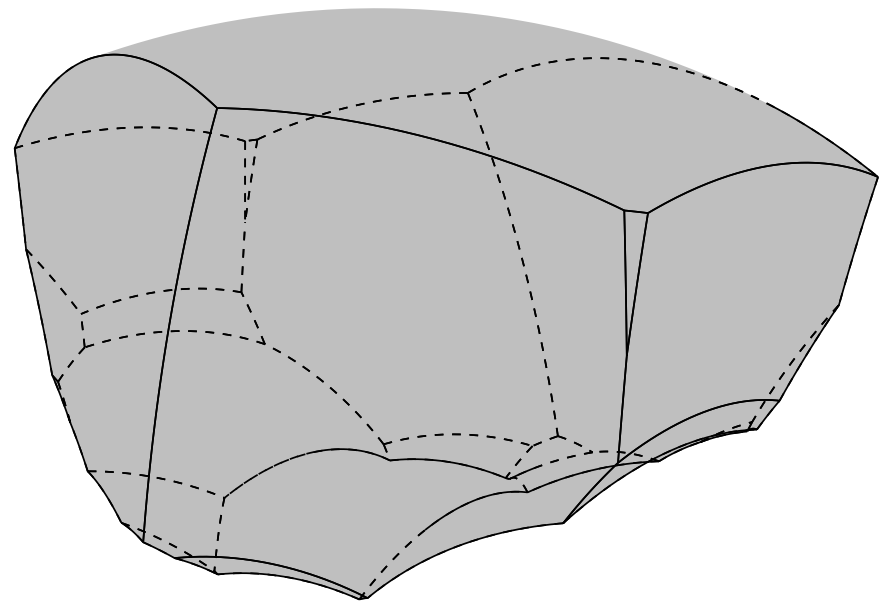

FIGURE 2.

3.3. The manifold has no manifold quotients. Here we establish the following theorem.

Theorem 3.1. $M$ has volume $v_{0}$ and does not properly cover any hyperbolic 3manifold.

This theorem is also a direct consequence of the fact that $M$ is arithmetic. For, as will be shown in the proof, $M$ has volume that of the regular ideal simplex in $\mathbf{H}^{3}$, and the minimal volume of an arithmetic hyperbolic 3-manifold is approximately $0.94 \ldots$ ([4]). However, we give a direct proof as it will illustrate the techniques to be applied to some of the other exceptional groups. 
The proof is similar to the proof of Lemma 8 of [12]. Before embarking on the proof, we make some preliminary observations.

From above, $\operatorname{Ram} A$ consists of the places $\mathcal{P}_{4}$ and $\mathcal{P}_{3}$ associated to the primes 2 and $\sqrt{-3}$ of $\mathbf{Q}(\sqrt{-3})$. As can be checked, the type number of this algebra is 1. Hence up to conjugacy in $\operatorname{PGL}(2, \mathbf{C})$ there is a unique group $\Gamma_{\mathcal{O}}$ arising from maximal orders $\mathcal{O}$ of $A$. From the volume formula in $\S 2.3$ we deduce

$$
\operatorname{Vol}\left(\mathbf{H}^{3} / \Gamma_{\mathcal{O}}\right)=\frac{2 \pi^{2} \zeta_{\mathbf{Q}(\sqrt{-3})}(2) 3^{\frac{3}{2}}\left(\frac{3}{2}\right)}{\left(4 \pi^{2}\right)^{2}} .
$$

Furthermore using the fact that the volume $v_{0}$ of a regular ideal simplex in $\mathbf{H}^{3}$ is given by

$$
\frac{3^{5 / 2} \zeta_{\mathbf{Q}(\sqrt{-3})}(2)}{2 \pi^{2}}
$$

we obtain the following.

Lemma 3.2. $\operatorname{Vol}\left(\mathbf{H}^{3} / \Gamma_{\mathcal{O}}\right)=\frac{v_{0}}{8}$.

Proof of Theorem 3.1. Note that from above since $H_{1}(M, \mathbf{Z})$ is finite, $M$ cannot cover a non-orientable hyperbolic 3-manifold. Thus it suffices to rule out orientable manifolds in the commensurability class determined by the algebra $A$ above.

We aim to locate $M$ in the commensurability class defined by $A$. Let $\Gamma$ denote the faithful discrete representation of $\pi_{1}(M)$ defined above. As discussed in $\S 3.1, M$ covers the orbifold $Q=\mathbf{H}^{3} / \Gamma_{\mathcal{O}}$. By using a volume estimate for $M$ together with Lemma 3.2 we must have that $M$ is an 8 -fold cover of $Q$, and so has volume $v_{0}$. Also as noted above this is the unique orbifold of this volume in the commensurability class of $M$. We shall show that the only minimal orbifold that $M$ can cover is $Q$. For then if $M$ covers a hyperbolic 3-manifold $N$ say, $N$ must also cover $Q$, and the degree of the cover is at most 4 . However from $\S 3.1$, the group $\Gamma_{\mathcal{O}}$ contains a dihedral group of order 8 , so any manifold cover of $Q$ must have degree at least 8 . This rules out a manifold cover $N$, and completes the proof of the theorem.

To show that $M$ does not cover any other minimal orbifold we use Borel's description of maximal groups in the commensurability class given in $\S 2$. This will involve a simple analysis of primes of small norm in $\mathbf{Q}(\sqrt{-3})$ together with the volume formula for $\Gamma_{S, \mathcal{O}}$ given in $\S 2.3$. Thus, assume that $\Gamma$ is a subgroup (up to conjugacy) of some maximal group $\Gamma_{S, \mathcal{O}}$. Then the volume formula in $\S 2.3$ gives

$$
\operatorname{Vol}\left(\mathbf{H}^{3} / \Gamma_{S, \mathcal{O}}\right)=\frac{v_{0}}{8} \cdot \frac{\prod_{\mathcal{P} \in S}(N \mathcal{P}+1)}{2^{m}},
$$

where $0 \leq m \leq|S|$. Also recall $S$ consists of places different from $\mathcal{P}_{4}$ and $\mathcal{P}_{3}$ at which $A$ is ramified. Now 5 is inert in $\mathbf{Q}(\sqrt{-3})$ giving a place $\mathcal{P}_{25}$, and 7 splits as a pair of primes $\mathcal{P}_{7} \mathcal{P}_{7}^{\prime}$. We make some comments on the possibilities for $S$. Let $T=\left\{\mathcal{P}_{4}, \mathcal{P}_{3}, \mathcal{P}_{25}, \mathcal{P}_{7}, \mathcal{P}_{7}^{\prime}\right\}$

$S$ contains a place not in $T$. Since 11 is inert and 13 splits, the minimal possible norm of a place in this case is 13 . Thus if $\mathcal{P}$ is as stated, then the co-volume of $\Gamma_{S, \mathcal{O}}$ is at least $\frac{v_{0}}{8} \cdot 7$. Then $\Gamma$ having co-volume $v_{0}$ cannot be a subgroup of such a group.

$S$ contains $\mathcal{P}_{25}$. Arguing as above we get a volume estimate of at least $\frac{v_{0}}{8} \cdot 13$ which is larger than $v_{0}$, and hence $\Gamma$ cannot be a subgroup of such a $\Gamma_{S, \mathcal{O}}$. 
$S$ contains $\mathcal{P}_{7}$ or $\mathcal{P}_{7}^{\prime}$. By definition $N \mathcal{P}_{7}=N \mathcal{P}_{7}^{\prime}=7$, so that $S$ cannot contain both of $\mathcal{P}_{7}$ and $\mathcal{P}_{7}^{\prime}$, for again as above we get a co-volume estimate of $\frac{v_{0}}{8} \cdot 16$ which is greater than $v_{0}$. Thus these remarks together with the above imply that the only possibilities for $S$ (apart from $S=\emptyset$ which is dealt with by $\Gamma_{\mathcal{O}}$ ) are $S=\left\{\mathcal{P}_{7}\right\}$ and $S=\left\{\mathcal{P}_{7}^{\prime}\right\}$. The volume estimates in these cases give $\frac{v_{0}}{8} \cdot 4=v_{0} / 2$.

To rule out these cases as giving maximal groups for which $\Gamma$ is a subgroup we proceed as follows. From above if $\Gamma$ were a subgroup $\Gamma_{S, \mathcal{O}}$ the index is 1 or 2 . If the index is 1 , then $\Gamma_{S, \mathcal{O}}<\Gamma_{\mathcal{O}}$ and so $\Gamma_{S, \mathcal{O}}$ is not maximal. If the index is 2 , then $\Gamma$ is normal in $\Gamma_{S, \mathcal{O}}$. But from $\S 3.1, \Gamma$ is normal in $\Gamma_{\mathcal{O}}$ and so $\Gamma_{\mathcal{O}}$ is the normalizer of $\Gamma$ in $\operatorname{PGL}(2, \mathbf{C})$, since it is a maximal group. Thus as above we deduce that $\Gamma_{S, \mathcal{O}}<\Gamma_{\mathcal{O}}$ and so $\Gamma_{S, \mathcal{O}}$ is not maximal.

Remark 3.3. It follows from the presentation of $\Gamma_{\mathcal{O}}$ that $M$ is the unique arithmetic manifold of this volume in the commensurability class, since all other index 8 subgroups have elements of finite order.

\section{OTHER EXCEPTIONAL MANIFOLDS}

There are six other exceptional manifolds $X_{1}, \ldots, X_{6}$ mentioned in [6]. Following the methods in $\S 3$, and using the approximations given in [6], it is possible to construct (as suggested by $\S 3$ of [6]) closed arithmetic hyperbolic 3-manifolds associated to five of these - the exception being $X_{3}$. Hence the uniqueness statement conjectured for the remaining five are susceptible to attack by the methods used above. We now discuss this.

Two of these, $X_{5}$ and $X_{6}$, are homeomorphic by an orientation reversing isometry. To see this, we note that both of these have $R^{\prime}=1$, and their $L^{\prime}$ and $D^{\prime}$ parameters are negative conjugates of each other. Complex conjugation of all parameters is an orientation reversing isometry and it is readily seen from our formulae above that, when $R^{\prime}=1$, changing the sign of $L^{\prime}$ or $D^{\prime}$ (or both) changes the signs of two traces, resulting in the same representation into $\operatorname{PSL}(2, \mathbf{C})$. Hence, we drop consideration of $X_{6}$.

The remaining four, however, have significantly larger volume than Vol3 and hence are more difficult to correlate with previously-known manifolds. In addition, the index of each of these in the corresponding group of minimal co-volume in the commensurability class is 24 or 48 (instead of 8), greatly complicating the analysis.

As one example of this complication, we mention that the group of minimal co-volume commensurable with $\pi_{1}\left(X_{5}\right)$ (in which $\pi_{1}\left(X_{5}\right)$ has index 48) has 140 conjugacy classes of index- 48 torsion-free subgroups and 24 conjugacy classes of index-24 torsion-free subgroups. The problem of establishing that $X_{5}$ has no manifold quotients then becomes one of identifying the conjugacy class of $\pi_{1}\left(X_{5}\right)$ inside this maximal group and then verifying that this class does not admit a conjugacy class of index-24 supergroups.

Hence we limit ourselves here to a few comments and a summary of some of the relevant arithmetic data on these manifolds. First, note that each of these manifolds has first betti number equal to zero, so that only orientable quotients need be considered. Second, note that here there are other maximal groups to be considered, in addition to the group of minimal co-volume in the commensurability class. These maximal groups have an alternative description (in addition to Borel's notation mentioned above) as normalizers of Eichler orders (intersections of two 
TABLE 1.

\begin{tabular}{|l|c|c|c|c|}
\hline & $X_{1}$ & $X_{2}$ & $X_{4}$ & $X_{5}$ \\
\hline Inv. Trace Field & $\begin{array}{c}t^{4}-2 t^{3}-t^{2} \\
+2 t-1\end{array}$ & $t^{2}+1$ & $t^{3}-t-2$ & $\begin{array}{c}t^{3}-t^{2} \\
-t-1\end{array}$ \\
\hline Ramification Set & none & $\mathcal{P}_{2}, \mathcal{P}_{5}$ & $\mathcal{P}_{2}$ & $\mathcal{P}_{2}$ \\
\hline $\begin{array}{l}\text { Norm of } \\
\text { Order Disc. }\end{array}$ & 217 & 20 & 176 & 16 \\
\hline $\begin{array}{l}\text { Index in } \\
\text { Max. Group }\end{array}$ & 48 & 24 & 24 & 48 \\
\hline Volume & 4.116968 & 3.663862 & 7.517689 & 3.177293 \\
\hline$H_{1}$ & $\mathbf{Z}_{7} \oplus \mathbf{Z}_{7}$ & $\mathbf{Z}_{4} \oplus \mathbf{Z}_{12}$ & $\mathbf{Z}_{4} \oplus \mathbf{Z}_{12}$ & $\mathbf{Z}_{4} \oplus \mathbf{Z}_{4}$ \\
\hline Eichler Orders & $\left\{\mathcal{P}_{7}\right\}$ & none & $\left\{\mathcal{P}_{2}^{\prime}\right\},\left\{\mathcal{P}_{11}\right\}$ & none \\
\hline Trace Triple & $2 t^{3}-3 t^{2}-4 t+1$ & $-2 t-2$ & $t^{2}-2 t-1$ & $2 t^{2}-2 t-2$ \\
& $2 t^{3}-3 t^{2}-4 t+1$ & $-2 t-2$ & $t^{2}-2 t-1$ & $2 t^{2}-2 t-2$ \\
& $-5 t^{3}+9 t^{2}+8 t-8$ & $6 t$ & $2 t^{2}+2 t-6$ & $-2 t^{2}+4 t$ \\
\hline
\end{tabular}

maximal orders). Some of these may be ruled out by various means, however, which reduces the number of Eichler orders which must be computed.

For example, in the case of $X_{1}$, the index of $\pi_{1}\left(X_{1}\right)$ inside the minimal co-volume group is 48. Denote this minimal volume by $V$. Then any other maximal group containing $\pi_{1}\left(X_{1}\right)$ would have a volume $K V$ where $K$ is a "factor" of 48 ( $K$ need not be an integer, but it is an integer divided by a power of 2 ). Thus, the norm of any prime ideals in the corresponding set $S$ must be one of $\{2,3,5,7,11,23,31,47\}$. Not all of these norms actually exist among the prime ideals of the invariant trace field. This leaves only $\{7,23,31,47\}$ to consider.

Furthermore, we need only consider the combinations of distinct primes $\mathcal{P}$ in which the product of $(N \mathcal{P}+1) / 2$ is less than or equal to 24 . This leaves only single-element sets of these four norms to consider.

We can additionally apply Lemma 2.2.3 of 4 to rule out 31 on the grounds that the normalizer of the corresponding Eichler order must contain 3-torsion, and thus any torsion-free subgroups would have the same (or larger) volume as $X_{1}$.

Finally, we rule out any combinations containing prime ideals that do not divide the discriminant of the order generated by the trace triple of $\pi_{1}\left(X_{1}\right)$ since such primes cannot appear in the discriminant of an Eichler order containing $\pi_{1}\left(X_{1}\right)$ (see for example [13, Cor. 4.8, p. 25).

Taking all of this together, we find only one Eichler order, corresponding to $S=\left\{\mathcal{P}_{7}\right\}$ (where $\mathcal{P}_{7}$ is the unique prime of norm 7 in the invariant trace field) which needs to be considered in addition to the maximal order in checking whether or not $X_{1}$ has a nontrivial manifold quotient.

Table 1summarizes the relevant arithmetic data for the four other unique arithmetic exceptional manifolds. In this table, the fields are specified by giving a polynomial, adjoining one root of which to $\mathbf{Q}$ gives the field, prime ideals are given in the form $\mathcal{P}_{n}$, referring to an ideal of norm $n$, volumes are given to seven significant figures without rounding, and Eichler orders are specified by giving the corresponding set $S$ of prime ideals. In the description of the Eichler orders of $X_{4}$, the notation $\mathcal{P}_{2}^{\prime}$ refers to the unique ideal of norm 2 other than the one that ramifies 
the algebra. We note also that all four of these algebras have type number 1 (hence a unique conjugacy class of maximal orders).

Remark 4.1. The results of [4] can be used to reduce the degree of possible covering of $X_{i} \rightarrow N$, where $N$ is a conjectural closed hyperbolic 3-manifold quotient. For instance for $X_{2}$ the only possible degrees are 2 and 3, reducing the enumeration of subgroups of finite index to index 12 and 8.

\section{ACKNOWLEDGEMENT}

We are most grateful to D. Gabai, R. Meyerhoff and N. Thurston for correspondence on the manifolds arising in their work.

\section{REFERENCES}

1. H. Bass, Groups of integral representation type, Pacific J. Math. 86 (1980), 15-51. MR 82c:20014

2. A. Borel, Commensurability classes and volumes of hyperbolic 3-manifolds, Ann. Scuola Norm. Sup. Pisa 8, (1981), 1-33. MR 82j:22008

3. T. Chinburg and E. Friedman, The smallest arithmetic hyperbolic 3-orbifold, Invent. Math. 86, (1980), 507-527. MR 88a:22022

4. T. Chinburg, E. Friedman, K. N. Jones and A. W. Reid, The arithmetic hyperbolic 3manifold of smallest volume, Ann. Scuola Norm. Sup. Pisa, to appear.

5. D. Gabai, The geometric and topological rigidity of hyperbolic 3-manifolds, J. A. M. S. 10 (1997), 37-74. MR 97h:57028

6. D. Gabai, R. Meyerhoff and N. Thurston, Homotopy hyperbolic 3-manifolds are hyperbolic, Preprint.

7. H. M. Hilden, M.-T. Lozano and J.-M. Montesinos, A characterization of arithmetic subgroups of $\mathrm{SL}(2, \mathbf{R})$ and $\mathrm{SL}(2, \mathbf{C})$, Math. Nachr. 159 (1992), 245-270. MR 94i:20088

8. K. N. Jones and A. W. Reid, Computational methods in arithmetic Kleinian groups, in preparation.

9. C. Maclachlan and A. W. Reid, Commensurability classes of arithmetic Kleinian groups and their Fuchsian subgroups, Math. Proc. Camb. Phil. Soc. 102 (1987), 251-257. MR 88j:20040

10. W. D. Neumann and A. W. Reid, Arithmetic of hyperbolic 3-Manifolds, Topology '90, Proc. of Low-dimensional Topology Conference, Ohio State Univ., De Gruyter (1991), 273310. MR 94c:57024

11. A. W. Reid, A note on the trace-fields of Kleinian groups, Bull. L. M. S. 22 (1990), 349-352. MR 91d:20056

12. A. W. Reid, A non-Haken hyperbolic 3-manifold covered by a surface bundle, Pacific J. Math. 167 (1995), 163-182. MR 95m:57025

13. M.-F. Vignéras, Arithmétique des algèbres de Quaternions, L.N.M. 800, Springer Verlag, Berlin, 1980. MR 82i:12016

Department of Mathematical Sciences, Ball State University, Muncie, Indiana 47304

E-mail address: kerryj@math.bsu.edu

Department of Mathematics, University of Texas, Austin, Texas 78712

E-mail address: areid@math.utexas.edu 